\title{
A GROUP THEORETIC CRITERION FOR PROPERTY FA
}

\author{
MARC CULLER AND KAREN VOGTMANN
}

(Communicated by James West)

\begin{abstract}
We give group-theoretic conditions on a set of generators of a group $G$ which imply that $G$ admits no non-trivial action on a tree. The criterion applies to several interesting classes of groups, including automorphism groups of most free groups and mapping class groups of most surfaces.
\end{abstract}

\section{INTRODUCTION}

Following Serre [9], a group $G$ is said to have property FA if every action of $G$ on a tree is trivial. (An action on a tree is trivial if there is a point of the tree fixed by every element of $G$.) Serre proved that $S L(3, \mathbb{Z})$ has property FA. Margulis and Tits showed that subgroups of finite index in simple Chevalley groups of rank $\geq 2$ over a ring of integers $A$ also have property FA [10, Remark 4.3(b)]. Later, Bogopolski showed that the same is true for automorphism groups of free groups of rank at least three [4].

In this note we give group-theoretic conditions on a set of generators of a group $G$ which imply that $G$ has property FA. To illustrate our theorem, we give very simple proofs of property FA for several classes of groups, including special linear groups of rank at least 3, groups of automorphisms of free groups of rank at least 3 , and mapping class groups of most surfaces.

Serre's arguments actually imply that $S L(3, \mathbb{Z})$ has property $F \mathbb{R}$, which is defined to mean that every action on an $\mathbb{R}$-tree has a fixed point. In proving that a group $G$ has property $\mathrm{FR}$ one typically shows that any non-trivial action must have an invariant line upon which the group acts by translations. The existence of such an invariant line implies that $G$ admits a non-trivial homomorphism to $\mathbb{R}$. If the abelianization of $G$ is finite there are no non-trivial homomorphisms to $\mathbb{R}$, so that any action of $G$ must have a fixed point.

We will say that $G$ has property $A \mathbb{R}$ if every non-trivial action of $G$ on an $\mathbb{R}$ tree has an invariant line upon which $G$ acts by translation. Our main theorem gives a criterion for a group to have property $A \mathbb{R}$. The groups listed above satisfy our conditions and also have finite abelianizations. For $n \geq 5$ the $n$-strand braid groups satisfy our criterion and hence have property $\mathrm{A} \mathbb{R}$, although they do admit non-trivial homomorphisms to $\mathbb{R}$ and hence do not have property FA. G. Levitt has pointed out that results of Bieri-Neumann-Strebel [3] and Brown [5] imply that a

Received by the editors May 31, 1994.

1991 Mathematics Subject Classification. Primary 20E08; Secondary 20F28, 20F36, 05C05.

Key words and phrases. Property FA, group actions on trees.

Both authors are partially supported by the National Science Foundation. 
group with property $A \mathbb{R}$ must have a finitely generated commutator subgroup (see Remark 2.6).

\section{Characteristic subtrees}

We assume throughout this section that $G$ is a group acting by isometries on an $\mathbb{R}$-tree $X$. For background material on groups acting on $\mathbb{R}$-trees, we refer the reader to [9] and [1] or [6].

Given any element $g$ of $G$ we denote by $C_{g}$ the characteristic subtree of $g$. If $g$ is elliptic, $C_{g}$ is the subtree of $X$ fixed by $g$; if $g$ is hyperbolic, $C_{g}$ is the translation axis of $g$, also called $A_{g}$. The following simple observation will be useful.

Lemma 1.1. Let $g$ and $h$ be commuting elements of $G$, with $h$ hyperbolic. Then $C_{g}$ contains $A_{h}$.

Proof. For any $g$ and $h$ in $G$, we have $C_{h g h^{-1}}=h C_{g}$. If $g$ and $h$ commute, this says that $C_{g}$ is invariant under $h$. Any $h$-invariant subtree must contain the axis of $h$. $\square$

Minipotent words. A word in the letters $\left\{g^{ \pm 1}, h^{ \pm 1}\right\}$ is minipotent if it has one of the two forms

$$
g^{\epsilon_{1}} h^{\epsilon_{2}} \cdots g^{\epsilon_{2 n-1}} h^{\epsilon_{2 n}} \text { or } h^{\epsilon_{1}} g^{\epsilon_{2}} \cdots h^{\epsilon_{2 n-1}} g^{\epsilon_{2 n}},
$$

where $\epsilon_{i} \in\{ \pm 1\}$ for $i=1, \ldots, n$.

Proposition 1.2. Let $g$ and $h$ be elements of $G$, and let $w \in G$ be represented by a minipotent word in $g$ and $h$. If $C_{g} \cap C_{h}=\emptyset$, then $w$ is hyperbolic, and its axis contains the bridge from $C_{g}$ to $C_{h}$.

Proof. Let $[p, q]$ denote the bridge from $C_{g}$ to $C_{h}$. Let $P$ denote the subtree of $X$ consisting of all $x$ such that the geodesic $[x, q]$ contains $p$, and let $Q$ be the subtree consisting of all $x$ such that $[x, p]$ contains $q$. Note that $P$ and $Q$ are disjoint, that $g$ and $g^{-1}$ map $X-P$ into $P$ and that $h$ and $h^{-1}$ map $X-Q$ into $Q$. It follows by induction that if the minipotent word which represents $w$ begins with $g^{ \pm 1}$, then $w$ maps $X-Q$ into $P$, and that if the word begins with $h^{ \pm 1}$, then $w$ maps $X-P$ into $Q$. If $m$ is a point in the interior of $[p, q]$, then the two segments $\left[w^{-1} m, m\right]$ and $[m, w m]$ meet only at $m$ and their union contains $[p, q]$. It follows that $w$ is hyperbolic and its axis contains $[p, q]$.

Corollary 1.3. Let $g$ and $h$ be elements of $G$, and suppose that there exists an element $w$ of $G$ which commutes with either $g$ or $h$ and is represented by a minipotent word in $g$ and $h$. Then $C_{g} \cap C_{h} \neq \emptyset$.

Proof. Assume that $C_{g} \cap C_{h}=\emptyset$. Then Proposition 1.2 implies that $w$ is hyperbolic and that its axis contains the bridge from $C_{g}$ to $C_{h}$. In particular the axis of $w$ is not contained in either $C_{g}$ or $C_{h}$. This contradicts Lemma 1.1.

\section{Commutators.}

Lemma 1.4. If $h$ is hyperbolic with axis $A_{h}$, and $g \in G$ with $[g, h] A_{h}=A_{h}$, then $g A_{h}=A_{h}$.

Proof. We have $g h g^{-1} A_{h}=g h g^{-1} h^{-1} A_{h}=A_{h}$, so $A_{h}$ is the axis for the hyperbolic element $g h g^{-1}$. But the axis for $g h g^{-1}$ is $g A_{h}$, giving $g A_{h}=A_{h}$. 
Corollary 1.5. If $g$ and $h$ are hyperbolic, and $h$ commutes with $[g, h]$, then $A_{g}=$ $A_{h}$.

Proof. By Lemma 1.1, $A_{h}$ is invariant under $[g, h]$. Thus $A_{h}$ is invariant under $g$. The only line which is invariant under the action of a hyperbolic isometry is its axis.

Corollary 1.5 together with Corollary 1.3 are all we need for examples where the generators are all conjugate, e.g. Aut $\left(F_{n}\right)$, special linear groups, mapping class groups and braid groups. In general we need to consider the possibility that some generators are hyperbolic and some are elliptic. For this, we consider a special class of minipotent words which are iterated commutators.

For $x, y \in G$, we define $\left[x, y^{(k)}\right]$ inductively by

$$
\left[x, y^{(0)}\right]=x \quad \text { and } \quad\left[x, y^{(k)}\right]=\left[\left[x, y^{(k-1)}\right], y\right] \text {. }
$$

Proposition 1.6. Let $h$ be hyperbolic and $g \in G$. If there exists an integer $r>0$ such that $\left[g, h^{(r)}\right]=1$, then $g A_{h}=A_{h}$. If $g$ is elliptic and there exists an integer $s>0$ such that $\left[h, g^{(s)}\right]=1$, then $g$ fixes $A_{h}$.

Proof. By Lemma 1.1, $C_{\left[g, h^{(r-1)}\right]}$ contains $A_{h}$, i.e., $A_{h}$ is invariant under $\left[g, h^{(r-1)}\right]$. Applying Lemma 1.4 inductively shows $A_{h}$ is invariant under $g$. If $g$ is elliptic and does not fix $A_{h}$, then $g$ must act on $A_{h}$ as a reflection. The second conclusion follows from the fact that the relation $\left[h, g^{(s)}\right]=1$ does not hold in an infinite dihedral group generated by a reflection $g$ and a translation $h$; in fact, $\left[h, g^{(s)}\right]$ acts on $A_{h}$ as translation by $2^{s}|h|$.

\section{PROPERTY AR}

Our criterion for a group to have property $A \mathbb{R}$ is expressed in terms of a graph associated to a given set of generators for the group. We will regard an edge of a graph as being represented by a pair of oppositely oriented directed edges. We fix a group $G$ and a set of generators $S=\left\{s_{1}, \ldots, s_{n}\right\}$ for $G$.

The graph $\Delta$. Define $\Delta(G, S)$ to be the graph with vertex set $S$ and an edge between $s_{i}$ and $s_{j}$ whenever there is a minipotent word in $s_{i}$ and $s_{j}$ which commutes with either $s_{i}$ or $s_{j}$. (In particular, if $s_{i}$ and $s_{j}$ commute, they are joined by an edge, since they each commute with their (trivial) commutator.)

We will need the following criterion from [9] for the existence of a global fixed point for an action of a group $G$ on an $\mathbb{R}$-tree $X$. (Serre's proof is given for trees, but works equally well for $\mathbb{R}$-trees.)

Serre's Lemma. Let $G$ be generated by the finite set of elements $s_{1}, \ldots, s_{m}$, so that the $s_{j}$ and the $s_{i} s_{j}$ are all elliptic. Then $G$ has a fixed point.

Proposition 2.1. Suppose that $G$ acts on an $\mathbb{R}$-tree so that each $s_{i}$ is elliptic. If $\Delta(G, S)$ is the complete graph on $S$, then $G$ has a fixed point.

Proof. Since $\Delta(G, S)$ is complete, each pair $\left\{s_{i}, s_{j}\right\}$ is connected by an edge. If $s_{i}$ and $s_{j}$ are elliptic, Corollary 1.3 then implies that $s_{i} s_{j}$ is also elliptic. Thus the proposition follows from Serre's Lemma. 
The graph $\Delta^{\prime}$. Recall that the iterated commutators $\left[s_{i}, s_{j}^{(k)}\right]$ are minipotent, for $k>0$. If $\left[s_{i}, s_{j}^{(k)}\right]$ commutes with $s_{j}$ for some $k$, we say the directed edge of $\Delta(G, S)$ from $s_{i}$ to $s_{j}$ is distinguished. Define $\Delta^{\prime}(G, S)$ to be the subgraph of $\Delta(G, S)$ with vertex set $S$ and edge set consisting of the distinguished edges and their opposites.

A subset $V$ of $S$ is $\Delta^{\prime}$-connected if the subgraph of $\Delta^{\prime}(G, S)$ spanned by $V$ is connected.

A subset $V$ of $S$ is dense if for every $x \in S-V$, there is a distinguished edge from $x$ to some element of $V$ and a distinguished edge from some element of $V$ to $x$.

The following proposition gives a general criterion for a given action to have an invariant line upon which $G$ acts by translations.

Proposition 2.2. Suppose that $G$ acts on an $\mathbb{R}$-tree. If $S$ contains a nonempty, $\Delta^{\prime}$ connected, dense subset consisting of hyperbolic elements, then there is an invariant line upon which $G$ acts by translations.

Proof. Let $V$ be a non-empty $\Delta^{\prime}$-connected dense subset of $S$ consisting of hyperbolic elements. Since $V$ is $\Delta^{\prime}$-connected, Corollary 1.5 implies that all the elements of $V$ have the same axis $A$. Since $V$ is dense, Proposition 1.6 shows that $A$ is invariant under each hyperbolic element of $S$ and fixed by each elliptic element of $S$.

Group-theoretic criteria for property FA. The next theorem gives a grouptheoretic criterion, in terms of the generators $S$, for $G$ to have property AR. By a conjugacy class in $S$ we mean a non-empty subset which is the intersection of $S$ with a conjugacy class in $G$. Note that if one element of a conjugacy class is elliptic (hyperbolic), then so are all the other elements.

Theorem 2.3. Suppose that $\Delta(G, S)$ is the complete graph on $S$ and that each conjugacy class in $S$ contains a $\Delta^{\prime}$-connected dense subset. Then $G$ has property $\mathrm{AR}$.

Proof. If all elements of $S$ are elliptic, we apply Proposition 2.1 to conclude that the action is trivial. Otherwise there is a conjugacy class consisting of hyperbolic elements. Since this conjugacy class contains a connected dense subset, we may apply Proposition 2.2 to conclude that there is an invariant line on which $G$ acts by translations.

If the generators of $G$ are all conjugate, the criterion is more simply stated as follows:

Corollary 2.4. Suppose that all of the generators in $S$ are conjugate. If $\Delta(G, S)$ is complete and $\Delta^{\prime}(G, S)$ is connected, then $G$ has property $\mathrm{AR}$. If, in addition, the abelianization $H_{1}(G)$ is finite, then $G$ has property $\mathrm{F}$.

If the generators are not all conjugate, the following suffices.

Corollary 2.5. If $\Delta(G, S)$ is the complete graph on $S$ and every directed edge of $\Delta(G, S)$ is distinguished, then $G$ has property $\mathrm{A} \mathbb{R}$. If, in addition, the abelianization $H_{1}(G)$ is finite, then $G$ has property $\mathrm{FR}$.

Proof. Under these hypotheses $\Delta(G, S)=\Delta^{\prime}(G, S)$ is complete, and every vertex $s \in S$ is dense. 


\section{Commutator subgroups and property $A \mathbb{R}$.}

Remark 2.6. If $G$ is a finitely generated group, let $S(G)$ be the set of non-trivial homomorphisms from $G$ to $\mathbb{R}$, modulo homotheties, and let $\Sigma(G) \subset S(G)$ be the Bieri-Neumann-Strebel invariant. Theorem B1 of [3] states that the commutator subgroup $G^{\prime}$ is finitely generated if and only if $\Sigma(G)=S(G)$. By Corollary 7.4 of [5], the complement of $\Sigma(G)$ in $S(G)$ consists of homomorphisms associated to non-trivial actions of $G$ on $\mathbb{R}$-trees with a fixed end but no invariant line. If $G$ has property $\mathrm{A} \mathbb{R}$, then every non-trivial action has an invariant line; in particular $\Sigma(G)$ is all of $S(G)$, and $G^{\prime}$ is finitely generated.

\section{EXAMPLES}

Automorphisms of free groups. Let $F_{n}$ be the free group of rank $n \geq 3$, with generators $x_{1}, \ldots, x_{n}$. We show that the group $\operatorname{Aut}\left(F_{n}\right)$ of automorphisms of $F_{n}$ has property $\mathrm{FR}$, for $n \geq 3$. We first consider the index two subgroup $S A_{n}$ consisting of special automorphisms (an automorphism is special if the determinant of the induced automorphism of $\mathbb{Z}^{n}$ is equal to +1$)$.

For $i \neq j$ let $\rho_{i j}$ (respectively $\lambda_{i, j}$ ) be the automorphism which sends $x_{i}$ to $x_{i} x_{j}$ (respectively $x_{j} x_{i}$ ) and fixes $x_{k}$ for $k \neq i$. The $\rho_{i j}$ and $\lambda_{i, j}$ generate $S A_{n}$. If $i, j$ and $k$ are distinct, then

$$
\left[\rho_{i j}, \rho_{j k}\right]=\rho_{i k} \quad \text { and } \quad\left[\lambda_{i j}, \lambda_{j k}\right]=\lambda_{i k}
$$

It follows that the automorphisms $r_{i}=\rho_{i, i+1}, l_{i}=\lambda_{i, i+1}(1 \leq i \leq n-1)$ and $r_{n}=\rho_{n, 1}, l_{n}=\lambda_{n, 1}$ generate $S A_{n}$. Set $S=\left\{r_{1}, l_{1}, \ldots, r_{n}, l_{n}\right\}$.

The group $S A_{n}$ contains the alternating group on $n$ letters and the automorphisms which send exactly two generators to their inverses. Conjugating by appropriate such automorphisms shows that the elements of $S$ are all conjugate.

Let $s_{i}=r_{i}$ or $l_{i}$. It is easily checked that $s_{i}$ commutes with $\left[r_{j}, s_{i}\right]$ and $\left[l_{j}, s_{i}\right]$ unless $j \equiv i+1(\bmod n)$. Thus $\Delta^{\prime}(G, S)$ is the complete graph on $S$ (although not all directed edges are distinguished). In addition the $s_{i}$ are all commutators, so the abelianization $H_{1}\left(S A_{n}\right)$ is trivial. We now apply Corollary 2.4 to conclude that $S A_{n}$ has property $\mathrm{FR}$.

If a group has property $\mathrm{FR}$, then finite extensions and quotient groups also have property FRR. It follows that the full automorphism group $\operatorname{Aut}\left(F_{n}\right)$ has property $\mathrm{FR}$, as do the groups $\operatorname{Out}\left(F_{n}\right)$ of outer automorphisms of $F_{n}, G L(n, \mathbb{Z})$ and $S L(n, \mathbb{Z})$. Property FA for $S A_{n}$ was first proved by O. V. Bogopolski [4], by a quite complicated argument. For $S L(n, \mathbb{Z})$, a proof is given in Serre's book on trees [9].

Special and general linear groups. For any finitely-generated commutative ring $A$ and $n \geq 3$, let $E_{n}(A)$ denote the subgroup of $S L(n, A)$ generated by the elementary matrices $e_{i, j}(a)$, where $a$ is a generator of $A$. If we set $S=\left\{e_{i, i+1}(a) \mid 1 \leq\right.$ $i \leq n-1\} \cup\left\{e_{n, 1}(a)\right\}$, then $\Delta^{\prime}\left(E_{n}(A), S\right)$ is complete, all edges are distinguished, and all the generators are commutators, so by Corollary $2.5, E_{n}(A)$ has property FR. If $A$ satisfies Bass's stable range condition $S_{d}$ (see [2]) and $S K_{1}(A)$ is finite, then $E_{n}(A)$ has finite index in $S L(n, A)$ for $n \geq d+2$, so we may conclude that $S L(n, A)$ has property $\mathbb{F}$ under these conditions.

Now consider the subgroup $G_{N}$ of $S L(n, \mathbb{Z})$ generated by $S=\left\{s_{1}, \ldots, s_{n}\right\}$, where $s_{i}=e_{i, i+1}^{N}, 1 \leq i \leq n-1$, and $s_{n}=e_{n, 1}^{N}$. 
Note that $s_{i}$ commutes with $s_{j}$ unless $i-j \equiv \pm 1(\bmod n)$, and in that case, $\left[s_{i}, s_{j}\right]$ commutes with both $s_{i}$ and $s_{j}$, since $\left[e_{i, j}^{r}, e_{j, k}^{s}\right]=e_{i, k}^{r s}$. Thus $\Delta^{\prime}\left(G_{N}, S\right)$ is the complete graph on $S$, and each directed edge is distinguished. In addition, $H_{1}\left(G_{N}\right)$ is finite, since a power of each generator can be expressed as a commutator. Thus, by Corollary $2.5, G_{N}$ has property $\mathrm{FR}$.

As remarked by Serre [9], it follows from a theorem of Tits that $G_{N}$ has finite index in $S L(n, \mathbb{Z})$; since any subgroup of finite index in $S L(n, \mathbb{Z})$ contains $G_{N}$ for some $N$, this shows that every subgroup of finite index in $S L(n, \mathbb{Z})$ has property $\mathrm{FR}$.

Mapping class groups of surfaces of positive genus. Let $M_{g, r}$ be the mapping class group of a surface of genus $g>0$ with $r \geq 0$ boundary components. For a survey of results on mapping class groups, see [7].

For any surface of genus at least one, the group $M_{g, r}$ is generated by a finite set $S$ of Dehn twists along non-separating simple closed curves, such that any two of these curves are either disjoint or intersect transversally in a single point.

Dehn twists along disjoint curves commute. If $a$ and $b$ are curves which intersect transversally in a single point and if the associated Dehn twists are $t_{a}$ and $t_{b}$, then we have the relation $t_{a} t_{b} t_{a}=t_{b} t_{a} t_{b}$. Thus $t_{a}$ and $t_{b}$ commute with the minipotent word $\left(t_{a} t_{b}\right)^{3}$. This shows that $\Delta\left(M_{g, r}, S\right)$ is the complete graph on $S$.

Since any non-separating simple closed curve can be taken to any other by a homeomorphism of the surface, the Dehn twists in $S$ are all conjugate in $M_{g, r}$.

If the genus $g$ is equal to 1 , the mapping class group $M_{g, r}$ has a non-trivial action on a tree with no invariant line. If $g$ is at least two, enough generators commute to make $\Delta^{\prime}\left(M_{g, r}, S\right)$ connected. In addition, $H_{1}\left(M_{g, r}\right)$ is finite [8]. Thus by Corollary 2.4, $M_{g, r}$ has property $\mathrm{F} \mathbb{R}$ for $g \geq 2$.

Braid groups and mapping class groups of punctured spheres. The classical braid group $B_{n}$ has a generating set $S=\left\{\sigma_{1}, \ldots, \sigma_{n-1}\right\}$. These generators are all conjugate. The generators $\sigma_{i}$ and $\sigma_{j}$ commute when $0 \leq i, j \leq n-1$ and $|i-j| \geq 2$ and the relation

$$
\sigma_{i} \sigma_{i+1} \sigma_{i}=\sigma_{i+1} \sigma_{i} \sigma_{i+1}
$$

holds for $1 \leq i \leq n-2$. As in the preceding example of mapping class groups, we have that $\Delta\left(B_{n}, S\right)$ is the complete graph on $S$, and $\Delta^{\prime}\left(B_{n}, S\right)$ is connected if $n \geq 5$. However $B_{n}$ does admit non-trivial homomorphisms onto $\mathbb{Z}$. So we can only conclude that $B_{n}$ has property $\mathrm{AR}$, for $n \geq 5$. It can be seen from the presentation that each of the groups $B_{3}$ and $B_{4}$ admits a non-trivial action on a tree with no invariant line.

The mapping class group $M_{0}^{s}$ of a sphere with $s$ punctures (which may be permuted) is a quotient of the braid group $B_{s}$, so inherits property $A \mathbb{R}$ for $s \geq 5$. In addition, $H_{1}\left(M_{0}^{s}\right)$ is finite for all $s$, so $M_{0}^{s}$ has property $\mathbb{R}$ for $s \geq 5$. For $s=0$ or $1, M_{0}^{s}$ is trivial, and $M_{0}^{2} \cong \mathbb{Z} / 2 \mathbb{Z} ; M_{0}^{3}$ is generated by two elements of order two, whose product has order 3 , so that Serre's lemma applies directly to show that $M_{0}^{3}$ has property $\mathrm{FR}$. The group $M_{0}^{4}$ has a non-trivial action on a tree.

Nilpotent groups. Consider any finite set $S$ of generators of a nilpotent group $G$. It is immediate that $\Delta^{\prime}(G, S)$ is the complete graph on $S$, and that all directed edges in $\Delta^{\prime}(G, S)$ are distinguished. By Corollary 2.5, this gives another proof that nilpotent groups have Property $\mathrm{A} \mathbb{R}$. 


\section{REFERENCES}

1. R. Alperin and H. Bass, Length functions of group actions on $\Lambda$-trees, Combinatorial Group Theory and Topology (S. Gersten and J. Stallings, eds.), Ann. of Math. Stud., vol. III, Princeton Univ. Press, Princeton, NJ, 1986, pp. 265-378. MR 89c:20057

2. H. Bass, Algebraic K-theory, Benjamin, New York, 1968. MR 40:2736

3. R. Bieri, W. D. Neumann, and R. Strebel, A geometric invariant of discrete groups, Invent. Math. 90 (1987), 451-477. MR 89b:20108

4. O. V. Bogopolski, Arboreal decomposability of the group of automorphisms of a free group, Algebra i Logica 26 (1987), no. 2, 131-149.

5. K. Brown, Trees, valuations and the Bieri-Neumann-Strebel invariant, Invent. Math. 90 (1987), 479-504. MR 89e:20060

6. M. Culler and J. Morgan, Group actions on $\mathbb{R}-t r e e s$, Proc. London Math. Soc. (3) 55, 571-604. MR 88f:20055

7. N. V. Ivanov, Complexes of curves and the Teichmüller modular group, Russian Math. Surveys 42 (1987), no 3, 55-107. MR 88g:32040

8. D. Mumford, Abelian quotients of the Teichmüller modular group, J. Analyse Math. 18 (1967), 227-224. MR 36:2623

9. J.-P. Serre, Arbres, amalgames, $S L_{2}$, Asterisque 46 (1977). MR 57:16426

10. J. Tits, A theorem of Lie-Kolchin for trees, Contributions to Algebra: A Collection of Papers Dedicated to Ellis Kolchin (H. Bass, P. J. Cassidy, and J. Kovacic, eds.), Academic Press, New York, 1977, pp. 377-388. MR 58:28205

Department of Mathematics, University of Illinois at Chicago, 851 S. Morgan St., Chicago, Illinois 60607-7045

E-mail address: culler@math.uic.edu

Department of Mathematics, Cornell University, White Hall, Ithaca, New York 14853-7901

E-mail address: vogtmann@math.cornell.edu 